\title{
CUIDADO DE LA PERSONA CON DISCAPACIDAD MOTRIZ
}

\section{CARE OF THE PERSON WITH MOTOR DISABILITIES}

Recibido septiembre 2015 Aceptado octubre 2015 Publicado noviembre 2016

Correspondencia:

Calzada México Xochimilco \# 289, Colonia Arenal de Guadalupe. CP 14389. Delegación Tlalpan Teléfono: 59991000 Ext. 12220 shcorral@gmail.com

\section{Autores:}

Sandra Hernández-Corral

Subjefe de Educación e Investigación en Enfermería, Instituto Nacional de Rehabilitación

Ma. Guadalupe Ojeda-Vargas

Profesora de tiempo completo, Departamento de Enfermería y Obstetricia, División de Ciencias de la Salud e Ingenierías, Campus Celaya Salvatierra, Universidad de Guanajuato.

Palabras clave: Cuidado, discapacidad

Key words: Care, disability 


\section{RESUMEN}

En este artículo se describe el cuidado de la persona con discapacidad motriz, es por ello que se analliza el cuidado desde los enfoque teóricos de Leonardo Boff y Regina Vera Waldow para establecer una relación con los cuidados en rehabilitación proporcionados por el profesional de enfermería. Finalmente se reflexiona acerca de lo profundo del significado del cuidado, considerando que abarca más que solo intervención, es una forma de estar en el mundo en la que establecen a través de la con-vivencia y co-existencia relaciones transformadoras, generando crecimiento entre la persona que se cuida y la enfermera.

Palabras clave: Cuidado, discapacidad

\section{ABSTRACT}

In this article the care of people with mobility disabilities described, which is why care is analyzed from the theoretical approach of Leonardo Boff and Regina Waldow Vera to establish a relationship with the rehabilitation care provided by the nurse. Finally we reflect on the deep meaning of care, considering that encompasses more than one intervention, is a way of being in the world in establishing through-existence and co-existence transformative relationships, generating growth between caring person and nurse.

Key words: Care, disability

\section{INTRODUCCIÓN}

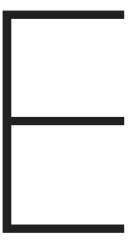

n su forma más antigua, Cura en latín se escribía coera, y era utilizada en un contexto de relaciones de amor y amistad. Expresaba la actitud de cuidado, de desvelo, de inquietud y preocupación por la persona amada o por un objeto de valor sentimental; en este sentido el cuidado sólo surge cuando la existencia de alguien tiene importancia para mí. Cuidado significa entonces, diligencia, celo, atención, delicadeza; es un modo de ser mediante el cual la persona sale de sí y se centra en el otro con desvelo y solicitud?

El cuidado es una forma de ser hombre, y tiene un significado a partir del propio hombre. Incluyendo comportamientos, actitudes, valores y principios que son vividos por las personas en determinadas circunstancias, sin embargo se refiere al ser. El ser humano nace con un potencial de cuidado, lo que significa que todas las personas son capaces de cuidar, evidentemente esta capacidad será más o menos desarrollada, conforme a las circunstancias en que fuera ejecutada en las diversas etapas de la vida. ${ }^{2}$

Se reconoce que los seres humanos requieren de mayor cuidado para desarrollarse durante la infancia y la tercera edad, cuando se evidencia dependencia para realizar actividades de la vida diaria, tanto de tipo físico-social como mental. La enfermedad, la incapacidad y el sufrimiento son algunas de las circunstancias que causan vulnerabilidad, originando que los individuos requieran de cuidado.

Por su propia naturaleza, el término cuidado incluye dos significados básicos, íntimamente vinculados entre sí. El primero, la actitud de desvelo, de solicitud, de atención hacia el otro. El segundo, la actitud de preocupación y de inquietud, porque la persona que proporciona el cuidado se siente implicada y vinculado afectivamente con el otro. ${ }^{3}$ Por otra parte el cuidar implica un movimiento en dirección de algo o alguien que es motivo de interés o preocupación. El ejercicio de cuidar es una acción que posee una direccionalidad

Enf Neurol Vol. 15. No. 1 enero - abril 2016 
concreta y determinada; nos mueve y nos impulsa a hacer algo, se trata de una acción moral objetiva, para aliviar, satisfacer, ayudar, confortar y apoyar. ${ }^{4}$

El cuidado es más que un acto individual o que una virtud al lado de otras. Es un modo de ser; a saber, la forma en que las persona se estructura y realiza en el mundo con los otros. $\bigcirc$, mejor aún: es un modo-de-ser-en-tmundo que funda las relaciones que se establecen con todas las cosas. ${ }^{3}$

El modo-de-ser-en-el-mundo significa una forma de existir y de coexistir, de estar presente, de navegar por la realidad y de relacionarse con todas las cosas del mundo. En esa co-existencia y con-vivencia, en esa navegación y en ese juego de relaciones, el ser humano va construyendo su propio ser, auto-conciencia y su propia identidad, fundamentalmente hay dos maneras básicas de ser-en-el-mundo: trabajo y cuidado, éste último no se opone al trabajo, pero le confiere una tonalidad diferente, gracias al cuidado se deja de ver como objetos la naturaleza y todo lo que existe en ella. La relación no es sujeto-objeto es sujeto-sujeto, la relación no es de dominio sobre, sino de con-vivencia, no es pura intervención, sino inter-acción y comunicación. ${ }^{3}$

Abarca, además de procedimientos y actividades técnicas, acciones y comportamientos que favorecen no sólo el estar con, sino también el ser con. Ser implica más que simplemente estar con; estar con puede tener lugar de modo que no hay participación real que, no se caracteriza por ser verdadera presencia. Esta presencia real, cuerpo y alma significa ser con. Cuidar es un compromiso, es ayudar a otros a crecer en la promoción de su integridad y unidad, manteniendo su dignidad y su singularidad. Por lo tanto el cuidar es un proceso interactivo, que solo se establece en la relación con el otro. ${ }^{5}$

El momento de cuidar es considerado de tipo transformador, en el cual, ambos, el ser cuidado y el cuidador crecen. El primero presenta una actitud más positiva y serena frente a su experiencia con la enfermedad, incapacidad e inclusive la muerte, fruto de una tranquila y amistosa relación de confianza entre sus cuidadores. Es importante que el paciente, sea considerado y respetado como una persona singular.2

Este modo-de-ser-enel-mundo, en forma de cuidado, permite al ser humano vivir la experiencia fundamental de valor, de aquello que tiene importancia y cuenta definitivamente. No del valor utilitarista, sólo para su uso, sino del valor intrínseco de las cosas. A partir de ese valor sustantivo surge la dimensión de alteridad, de respeto, de sacralidad, de reciprocidad y de complementariedad; en este camino surgen resistencias y aparece la confusión, pero todo ello se supera por medio de una paciencia perseverante. En lugar de agresividad, hay convivencia amorosa, en vez de dominación, hay compañía afectuosa, al lado de y junto con el otro. ${ }^{3}$

El cuidar tiene una connotación de proporcionar y promover el bienestar de los otros; por tanto es una acción muy peculiar, cuyo propósito es algo o alguien que necesita la acción del cuidador, el que sufre, el que está necesitado y vulnerable. La vulnerabilidad es una condición que requiere de cuidado, el cuidador responde a una necesidad expresada o referida, hay un intercambio y el encuentro entre el cuidador y el ser cuidado es siempre transformadora. ${ }^{5}$

Cuidar de las cosa implica tener intimidad con ellas, sentirlas dentro, acogerlas, respetarlas, darles sosiego y reposo. Cuidar es entrar en sintonía con las cosas, auscultar su ritmo y estar en armonía con ellas. La razón analítico-instrumental abre camino a la razón cordial, al espíritu de la delicadeza y al sentimiento profundo. El centro ya no está utilizado por la razón sino por el sentimiento. ${ }^{3}$

\section{8}

MMN Why Enf Neurol Vol.15. No. 1 enero - abril 2016 
El gran desafío para el ser humano es combinar trabajo con cuidado. Estas dos realidades no se oponen, sino que se componen se limitan muluamente y, a la vez, se complementan. Juntas constituyen la experiencia humana en su integridad, por un lado, vinculada a la materialidad y por otro, a la espiritualidad. El error consiste en oponer una dimensión a la otra y no verlas como modo-de-ser del único y mismo ser humano. ${ }^{3}$

Es importante resaltar que antes de cuidar a otro hay que cuidar de uno mismo, a este respecto, Waldow refiere el cuidar de sí como el cuidado personal, es decir, en una dimensión sujeto-self. Este cuidar de sí incluye, el conocimiento de sí, de sus potencialidades, necesidades y limitaciones. Ese conocimiento favorece una mejor autoestima, confianza en sí y en la vida. Comprende también el cuidar de la salud, del espíritu, del intelecto, de su tiempo y del ocio. ${ }^{6}$

Por otro lado, el cuidar a otro significa velar por su autonomía, desarrollar sus capacidades y no oponerse o ir en contra de sus decisiones libres y responsables. No obstante existen circunstancias, tales como las relacionadas a la necesidad de cuidado, considerando que la autonomía del ser se encuentra también vulnerable. Estas circunstancias requieren un análisis de relación entre la acción de cuidar y los límites de la autonomía en la persona, pues la autonomía no es ilimitada, presenta diversos grados y registro de desarrollo, por lo tanto es dinámica y concreta. ${ }^{2}$

Por lo que al asumir el cuidado del otro, la persona cuidadora estará privando al otro de cuidarse y desencadenará una situación de dependencia. Esta idea no es fácil de aceptar considerando que generalmente es necesario hacer por el otro determinadas actividades y por un período específico, tal vez lo difícil sea saber cuándo dejar de hacer o proveer condiciones a la persona, de ayudarse o ayudarlo a buscar alternativas para sobrevivir de forma más independiente posible. ${ }^{2}$
La vulnerabilidad está directamente asociada al cuidar, la persona es vulnerable en todas sus esferas, en la esfera física el sujeto puede enfermar, suffir dolor y discapacidad, debido a ello requiere cuidado; en este sentido la discapacidad es considerada como un problema de salud pública, se estima una incidencia anual de aproximadamente 26,000 casos; esta es resultado de lesiones y enfermedades que producen un daño permanente que conducen a deficiencias de largo plazo de tipo físico, mental, intelectual o sensorial, que con frecuencia implican una disminución en la calidad de vida, limitación de la capacidad y restricción de las personas que la padecen?

Uno de los tipos de discapacidad más frecuentes es la motriz, en esta se agrupan las músculo-esqueléticas y las neuromotrices. Las primeras se refieren a la dificultad que enfrenta una persona para moverse, caminar, mantener algunas posturas, así como las limitaciones en habilidades manipulativas como agarrar o sostener objetos. Las neuromotrices son aquellas que dificultan la movilidad de algún segmento corporal a consecuencia de un daño neurológico, incluyendo las secuelas de traumatismos y de algunas enfermedades como la poliomielitis, las lesiones medulares y distrofia muscular. ${ }^{8}$

Las personas con este tipo de discapacidad requieren de rehabilitación, para ello es necesaria la utilización de acciones interdisciplinarias y un esfuerzo conjunto entre todos los profesionales y familiares, dentro y fuera de las instituciones, teniendo como objetivo común mejorar las funciones disminuidas o perdidas para preservar la capacidad de vivir de cada individuo involucrando las acciones de cuidar?

Los cuidados en rehabilitación son indispensables para recobrar la capacidad motriz de las personas, este proceso tiene un componente actitudinal por parte de los profesionales hacia ejercer la rehabilitación compartiendo con el binomio paciente-familia, la búsqueda de la calidad del desempeño de las

Enf Neurol Vol. 15. No. 1 enero - abril 2016 
actividades de la vida diaria para vivir con un mínimo de independencia y con dignidad. Por lo que la persona es el elemento clave de equipo de rehabilitación, ella es el centro del equipo y la que determina los resultados finales del proceso. La familia es incorporada al equipo y es reconocida como un sistema dinámico que participa como un apoyo continuo en la solución de problemas y aprende a realizar cuidados necesarios. ${ }^{10}$

La asistencia de enfermería en la rehabilitación tiene como principales objetivos fomentar la independencia en la persona o alcanzar su máxima independencia dentro de sus condiciones, promover el autocuidado y prepararla para una vida social y familiar de la mejor manera posible y con calidad. 17,2 Por lo antes mencionado las personas son orientadas durante la hospitalización, sobre la importancia del autocuidado en la higiene corporal, alimentación e hidratación, estableciendo conjuntamente metas dentro de su funcionalidad, enfocándose a la realización de las actividades de la vida diaria de la manera más independiente posible, es necesario que la enfermera rehabilitadora conozca los hábitos y estilos de vida de la persona en el contexto familiar. Sus intervenciones comprenden el autocuidado para la capacidad de vestir y desvestir, alimentarse, realizar la higiene personal y prevenir deformidades de las artiouladiones, complicaciones respiratorias y vasculares. 10.13

Cabe destacar la implementación de programas de reeducación de la eliminación vesical e intestinal de acuerdo a las condiciones funcionales, físicas-motoras, sociales y familiares, en los cuales se incluye al binomio persona-cuidador, estimulando la participación en los cuidados, reconociendo los límites y las capacidades de cada uno Una vez que se tiene como principios que la rehabilitación es un proceso con un inicio y un final, el alta hospitalaria no puede ser encarada como un evento más sino como un proceso. Las orientaciones del alta son realizadas durante toda la hospitalización y no solamente el día del egreso hospitalario. ${ }^{10}$

Es importante resaltar la incorporación del cuidador al proceso de rehabilitación de la persona con discapacidad, con la finalidad aprender y promover los cuidados relacionados con la higiene corporal, la movilidad física, cambios de posición, transferencias, alimentación, eliminación, prevención de accidentes y de úlceras por presión, más allá de los relacionados a la reintegración social y apoyo emocional. El cuidador es un elemento importante para ofrecer la seguridad al paciente tanto en la hospitalización como en la continuidad de los cuidados posteriores al egreso. ${ }^{14}$

Como se puede apreciar en los párrafos anteriores el proceso de rehabilitación implica que la enfermera o el cuidador le realicen procedimientos a la persona con discapacidad, esto no se consideraría cuidado a menos que se desarrollen con una actitud de desvelo, de inquietud, preocupación, celo, atención y delicadeza por la persona a la que se cuida; cabe destacar que es imprescindible que el cuidador identifique las resonancias del cuidado a través de ciertas actitudes como son: el amor, la justa medida, la amabilidad y la convivencialidad, las cuales favorecen el logro de los objetivos de la rehabilitación, considerando que el proceso es largo y es conveniente en todo momento mantener una relación sujeto-sujeto en la que no prive la autonomía pero sí la dominancia y en la que la con-vivencia y la inter-acción originen una relación transformadora que propicie el crecimiento del cuidador y el ser cuidado. 


\section{REFERENCIAS BIBLIOGRÁFICAS}

1. Boff L. El cuidado necesario. Madrid: Trotta; 2012.

2. Waldow VR, Borges RF. O processo de cuidar sob a perspectiva da vulnerabilidade. Rev Latino-Am. Enfermagem. 2008; 16(4): 765-777.

3. Boff L. El cuidado esencial. Ética de lo humano compasión por la tierra. Madrid: Trotta; 2002.

4. Waldow VR. Cuidar: expresión humanizadora de la enfermería. México: Nueva Palabra; 2006.

5. Waldow VR. Nuevas ideas sobre el cuidado. Aquichán. 2008; 8(1): 85-96.

6. Waldow VR. Cuidando de si, del otro, del todo. Rev Paraninfo Digital. 2012; 15. (acceso 07 de junio de 2014). Disponible en: http://www.index-f.com/para/n15/002po.php

7. Ibarra LG, Ibarra JC, Segura VH. Discapacidad y Salud. México: Trillas; 2009.

8. Instituto Nacional Estadística Geográlica e Informática. La persona con discapacidad en México. México: INEGl; 2004.
9. Faro ACM. Enfermagen em Reabilitação: ampliando os horizontes, legitimando o saber. Rev Esc Enferm USP. 2006; 40(1): 128-133.

10. Leite VBE, Faro ACM. O cuidar do enfermeiro especialista en reabilitação físico-motora. Rev Esc Enferm USP. 2005; 39 (1): 92-96.

11. Barajas CA, Hernández CS, Garcia JM. Nivel de de independencia del paciente con lesión medular y su relación con las intervenciones de enfermería. Enf Neurol Mex. 2010; 10(2): 77-80

12. Hernández CS, Acosta AA. etiquetas diagnosticas más frecuentes en personas con lesión medular en fase de rehabilitación. Enfermería Universitaria. 2009; 6(1): 50-53.

13. Perlini NMOG, Faro ACM. Cuidar de pessoa incapacitada por acidente vascular cerebral no domicilio: o fazer do cuidador familiar. Rev Esc Enferm USP. 2005; 39(2): 154-163.

14. Carvalho Z, Lima FG, Holanda KM, Silva GA. Pacientes com lesão raquimedular: experiênica de ensino-aprendizagem do cuidado para suas famílias. Esc Anna Nery Enferm.2006; 10(2) : 316-322. 